\title{
Análisis de los Parámetros de Color en Salchichas Frankfurt Adicionadas con Extracto Oleoso de Residuos de Chontaduro (Bactris Gasipaes)
}

\author{
Lina X. Pinzón-Zárate, José I. Hleap-Zapata* y Luís E. Ordóñez-Santos \\ Universidad Nacional de Colombia, Facultad de Ingeniería y Administración, Departamento de Ingeniería, \\ Sede Palmira. A.A. 237. Carrera 32 No. 12-00. Palmira. Valle del Cauca-Colombia. \\ (e-mail: Ixpinzonz@unal.edu.co; jihleapz@unal.edu.co; leordonezs@unal.edu.co) \\ * Autor a quien debe ser dirigida la correspondencia
}

Recibido Ene. 15, 2015; Aceptado Mar. 23, 2015; Versión final Jul. 6, 2015, Publicado Oct. 2015

\begin{abstract}
Resumen
Este trabajo tuvo como objetivo evaluar el uso de un extracto oleoso de residuos de chontaduro (Bactris gasipaes) en la coloración de salchichas Frankfurt. Se midieron los valores de luminosidad ( $L$ ), coordenadas $\mathrm{a}^{*} \mathrm{y} \mathrm{b}^{*}$, croma $(\mathrm{C})$ y tono $(h)$. Se utilizó un diseño experimental completamente al azar con tres repeticiones, con un total de seis tratamientos derivados de la inclusión de cinco niveles de adición del extracto y uno control. El incremento en el porcentaje de adición del extracto condujo al aumento de los valores de luminosidad $(L)$, coordenada $\mathrm{b}^{*}$, croma $(C)$ y tono $(h)$ de acuerdo con el aumento en el porcentaje de extracto adicionado y la disminución en el porcentaje de nitritos. La coordenada $a^{*}$, por el contrario presentó un comportamiento decreciente. La aplicación del extracto representa una alternativa favorable, pero no ideal, para la sustitución de nitritos.
\end{abstract}

Palabras clave: carotenoides, aditivos cancerígenos, nitritos, Bactris gasipaes, alimento saludable

\section{Color Parameters Analysis in Frankfurter Sausages with the Addition of Oily Extract of Peach Palm (Bactris gasipaes) by- Products}

\begin{abstract}
This study aimed to evaluate the use of an oily residue of peach palm extract (Bactris gasipaes) for coloring frankfurter sausages. Values of luminosity $(L)$, coordinates $a^{*}$ and $b^{*}$, chroma $(C)$ and hue $(h)$ were measured. A completely randomized design with three replications was used, with a total of six treatments derived from the inclusion of five levels of addition of the extract and one control. The increasing values of lightness $(L)$, coordinate $\mathrm{b}^{*}$, chroma $(\mathrm{C})$ and hue $(h)$ was caused by the increase in the percentage of extract added and the decrease in the percentage of nitrite. The coordinate $a^{*}$, by contrast showed a decreasing trend. The oily extract application is a favorable alternative but it is not ideal for the replacement of nitrites.
\end{abstract}

Keyword: carotenoids, carcinogenic additives, nitrites, Bactris gasipaes, healthy food 


\section{INTRODUCCIÓN}

El color de la carne o de los productos cárnicos es un importante atributo de la calidad, el cual influye en la aceptación de los mismos por parte de los consumidores. Es un parámetro importante en la medición sensorial de los productos cárnicos procesados (Marchesi et al., 2006), los cuales tienden a decolorarse durante el procesamiento y almacenamiento (Deda et al., 2007). El color en los productos cárnicos es el producto de reacciones bioquímicas entre los compuestos naturales de la carne, tales como la mioglobina, la hemoglobina y el oxígeno y la acción de agentes externos tales como los nitratos y nitritos (Boles y Pegg, 2010). El rojo brillante que presentan algunos productos comerciales es consecuencia de la nitrosilmioglobina, la cual se deriva de la reacción de la mioglobina con el óxido nítrico (NO) resultado de la acción de los nitratos y/o nitritos adicionados (Sebranec y Bacus, 2007; Eyiler y Oztan, 2011; Hermann et al., 2015).

Además de la capacidad para brindar color a los productos cárnicos, el nitrito actúa como conservante (Hermann et al., 2015) y tiene propiedades antioxidantes (Andrée, et al., 2010; Weiss et al., 2010), pero la reducción en su uso se ha convertido en una tendencia para la industria cárnica debido a que bajo condiciones apropiadas de $\mathrm{pH}$ y temperatura reacciona con aminas formando nitrosaminas y otras toxinas que pueden ser cancerígenas (Amate et al., 2010; Crews, 2014; Kurkubic et al., 2014).

Diferentes estudios epidemiológicos muestran asociaciones entre el consumo de carne roja procesada (con adición de nitritos) y el mayor riesgo de contraer cáncer colonorectal (Kuhnle et al., 2010), cáncer de páncreas (Larsson y Wolk, 2012), enfermedades cardiovasculares, entre otras (Rohmann et al., 2013).

El mercado de los aditivos obtenidos a partir de fuentes naturales, desde el siglo pasado ha crecido ampliamente, debido a los peligros potenciales de los aditivos alimentarios artificiales y a los beneficios encontrados en compuestos biológicamente activos (Solymosi et al., 2015). Los colorantes provenientes de fuentes naturales prometen ser una alternativa para reducir el uso de nitritos y otros aditivos que mejoran o mantienen el color de los productos cárnicos ya que presentan actividad antioxidante y antimicrobiana (Pangestuti y Kim, 2011), y brindan características sensoriales de color y sabor aceptables por los consumidores (Eyiler y Oztan, 2011; Moreira et al., 2012).

Los colorantes naturales son los obtenidos directamente de animales, vegetales o minerales (Chengaiah et al., 2010), modificaciones químicas de los mismos compuestos, o duplicados producidos sintéticamente de compuestos de origen natural. Los carotenoides son compuestos solubles en lípidos y son los principales pigmentos naturales vegetales que proporcionan el color amarillo, naranja y rojo natural de frutas, hortalizas, plantas, aves y animales marinos (Fernández et al. 2007; Strati y Oreopoulou, 2011).

Los productos cárnicos, como matrices biológicas, para la aplicación de colorantes naturales resultan ser muy adecuados. Dentro de estas aplicaciones se pueden encontrar el licopeno, el aditivo natural más estudiado, en los últimos años, como aditivo cárnico. Como fuente para la obtención del mismo se ha utilizado mayormente el jugo y la pasta de tomate. El efecto del licopeno en la calidad de la carne durante el almacenamiento y los efectos de la cáscara de tomate en los embutidos fermentados han hecho parte de estas investigaciones (Mercadante et al., 2010). También se ha utilizado el cártamo (Carthamus tinctorius L.) como colorante de salchichas cocidas (Hyun-Wook et al., 2015). Los extractos acuosos de Mirtus communis tienen un excelente potencial, no solo como exaltadores del color de las salchichas tipo Frankfurt, sino como protectores durante la vida útil del producto (Amensour et al., 2010). Algunos colorantes como el carmín, el beta-caroteno, la norbixina y la zeaxantina han sido igualmente utilizados (Mercadante et al., 2010).

Una fuente promisoria natural de carotenoides en el departamento del Valle del Cauca - Colombia es el chontaduro (Bactris gasipaes) (Rojas-Garbanzo, 2009). Este fruto pertenece a la familia de Arecaceae (Tamayo y Román, 2010; Restrepo et al., 2012) y ha sido cultivado y consumido desde hace 2000 años en Latinoamérica como alimento de alto valor nutricional (Giraldo et al., 2012). Su distribución natural se extiende desde Panamá hasta Bolivia en regiones con una altitud inferior a $1000 \mathrm{~m}$, precipitaciones anuales entre 2000 y $5000 \mathrm{~mm}$ y temperatura media anual de más de $22^{\circ} \mathrm{C}$. Hoy en día es ampliamente cultivado fuera de su región original (Leterme et al. 2005). Los frutos son de forma ovoide, achatada, cilíndrica o cónica de 2 a 7 centímetros de largo. El peso total de la fruta varía entre 20 y $100 \mathrm{~g}$, dependiendo de la variedad (Vargas y Argüelles, 2000). Se componen de un epicarpio delgado que cubre al fruto, la parte comestible está constituida por el mesocarpio que es pulposo de color amarillo a naranja claro, seco y harinoso. Cada fruto tiene una sola semilla cubierta de un endocarpio de color oscuro, cónica, dura y aceitosa, con una almendra blanca similar en sabor y textura al coco (Coccus nucifera) (Bogantes, 2012).

Actualmente, el chontaduro es considerado como una importante alternativa de cultivos alimentarios y es objeto de estudio en Colombia para su uso en la elaboración de alimentos. Contiene altos niveles de fibra, 
aminoácidos esenciales, grasas y almidones (Leterme et al., 2005) y alto contenido de carotenoides (1,1-22,3 $\mathrm{mg}$ por cada $100 \mathrm{~g}$ de pulpa) (Jatunov et al., 2010).

El fruto del chontaduro se comercializa principalmente con mínima transformación (sólo cocción) destinada al consumo humano. Tanto el consumo directo como el procesamiento generan grandes cantidades de residuos, no cuantificados a la fecha, que no han sido suficientemente estudiados, desaprovechando su potencial agroindustrial y además generando acumulaciones indeseables. Dichos residuos aumentan los costos para su disposición y conllevan a la creación de un problema ambiental, debido a los altos volúmenes de material desechado (Bolanho et al. 2015). Uno de los usos que se le dan a estos residuos es la elaboración de barras alimenticias energéticas (Aguilar, 2010). Se ha estudiado, igualmente, el contenido de compuestos fenólicos solubles totales, obteniendo resultados de $108 \pm 2,06 \mathrm{mg} / 100 \mathrm{~g}$ de residuo fresco (Contreras et al., 2011).

Considerando que la tendencia actual en el procesamiento de alimentos, es la elaboración de comidas saludables y la estandarización de procesos tecnológicos amigables con el medio ambiente, sin generación de subproductos, nace la necesidad de investigar en la transformación agroindustrial de materias primas de origen natural.

Esta investigación se llevó a cabo con el objetivo de aplicar un extracto oleoso de carotenoides obtenidos de los residuos de chontaduro (Bactris gasipaes) en la formulación de salchichas Frankfurt como colorante natural y analizar la sustitución parcial de los nitritos en las mismas mediante la medición de las coordenadas de color CIELab.

\section{MATERIALES Y MÉTODOS}

\section{Elaboración de las salchichas tipo Frankfurt}

La investigación se desarrolló en el Laboratorio de Tecnología de Carnes de la Universidad Nacional de Colombia - sede Palmira (Valle del Cauca, Colombia). Para la elaboración de las salchichas se utilizó carne de cerdo y rebanado (tocino sin piel) de calidad comercial. El extracto oleoso de carotenoides, obtenido a partir de residuos de chontaduro (Bactris gasipaes), conformados por exocarpio y restos de mesocarpio, fue desarrollado en investigaciones previas en el laboratorio de Tecnología de Frutas de la misma Universidad por Ordóñez-Santos et al. (2015). Los demás insumos y aditivos se adquirieron en supermercados y empresas del sector alimentario de las ciudades de Palmira y Cali y correspondieron a los aditivos tradicionalmente utilizados para productos alimenticios cárnicos embutidos y avalados por la legislación colombiana.

La formulación base para todos los experimentos en la elaboración de las salchichas se llevó a cabo de acuerdo a la Norma Técnica Colombiana NTC 1325 (ICONTEC, 2008) ajustando algunos valores, como se observa en la Tabla 1.

Tabla 1: Formulación base de las salchichas.

\begin{tabular}{|c|c|}
\hline Ingredientes & Porcentaje (\%) \\
\hline Carne: lomo de cerdo & 70 \\
\hline Grasa: rebanado de cerdo & 20 \\
\hline Hielo & 10 \\
\hline
\end{tabular}

Haciendo uso de cuchillos en acero inoxidable desinfectados, se cortaron por separado y en trozos pequeños, la carne y la grasa. Estas materias primas se trasladaron a un molino M121 Marca Javar ${ }^{\circledR}$ (Javar, Bogotá, Colombia) dotado de una rejilla de $6 \mathrm{~mm}$ de diámetro. Posteriormente, fueron homogenizados los ingredientes con la carne, la grasa y el hielo en un cúter Hobart 84181 - D (Corporation Troy, Ohio, USA). Una vez obtenida la emulsión, se inició el proceso de embutido en una embutidora EM 30 Javar $^{\circledR}$ (Javar, Bogotá, Colombia) utilizando fundas artificiales (Amicel ${ }^{\circledR}$ Alico S.A., Cali, Colombia) calibre 22. Las salchichas se porcionaron a una distancia de $12 \mathrm{~cm}$, correspondiendo a un peso aproximado de $60 \mathrm{~g}$ y permanecieron durante 15 minutos a $80^{\circ} \mathrm{C}$ de temperatura medida con un termómetro de aguja Chektemp HI 98501 (HANNA Instruments Ltda., Leighton Buzzard, Bedfordsshire, Inglaterra) en un tanque de escaldado para luego ser sometidas a un choque térmico con agua fría durante 10 minutos. Finalmente, se empacaron en una máquina selladora al vacío EGARVAC S.C.P. Basic B (Vacarisses, Barcelona, España) y se almacenaron a temperatura de refrigeración $\left(6 \pm 2^{\circ} \mathrm{C}\right)$.

Los aditivos e insumos utilizados en la elaboración de las salchichas Frankfurt se listan en la Tabla 2. 
Tabla 2: Ingredientes utilizados en la elaboración de las salchichas.

\begin{tabular}{|c|c|}
\hline Aditivos e insumos & $\begin{array}{c}\text { masa cárnica } \\
\text { g/kg }\end{array}$ \\
\hline Sal & 10,0 \\
\hline Azúcar & 2,0 \\
\hline Fosfatos & 4,0 \\
\hline Ajo & 4,0 \\
\hline Sabor salchicha & 10,0 \\
\hline Glutamato Monosódico & 1,0 \\
\hline Ácido ascórbico & 1,0 \\
\hline Cebolla & 0,5 \\
\hline Pimienta & 0,5 \\
\hline
\end{tabular}

Se elaboraron seis lotes de salchichas tipo Frankfurt, los valores de los carotenoides y del nitral utilizados en la formulación de cada lote se muestran en la Tabla 3.

Tabla 3: Cantidad de nitritos y carotenoides en cada lote de experimentación.

\begin{tabular}{|c|c|c|c|}
\hline Lote & Nitritos, $\mathrm{mg} / \mathrm{kg}$ & Extracto Oleoso, $\mathrm{ml} / \mathrm{kg}$ & Carotenoides Totales, $\mathrm{mg} / \mathrm{kg}$ extracto \\
\hline 1 & 200 & 0 & 0,00 \\
\hline 2 & 180 & 9 & 2,61 \\
\hline 3 & 160 & 19 & 5,51 \\
\hline 4 & 140 & 29 & 8,41 \\
\hline 5 & 120 & 42 & 12,18 \\
\hline 6 & 100 & 97 & 28,13 \\
\hline
\end{tabular}

El máximo valor agregado de nitral $(200 \mathrm{mg} / \mathrm{kg})$ se determinó con base en lo estipulado por la Norma Técnica Colombiana NTC 1325. Para la cantidad mínima adicionada $(100 \mathrm{mg} / \mathrm{kg})$ se tuvo en cuenta lo reportado por Bazán-Lugo et al. (2011) quienes encontraron que valores inferiores pueden afectar la calidad microbiológica del alimento y por lo tanto su estabilidad durante el almacenamiento. Por su parte el valor máximo de extracto oleoso adicionado ( $97 \mathrm{ml} / \mathrm{kg}$ con un contenido de carotenoides totales de $28,13 \mathrm{mg}$ por $\mathrm{kg}$ de extracto) se estableció experimentalmente como la máxima cantidad posible agregada sin afectar la estabilidad de la emulsión.

\section{Medición de las coordenadas de color CIELab}

En un colorímetro marca Konica Minolta, Modelo: CR-400, empleando el iluminante D65 y el observador de $2^{\circ}$ (equipo calibrado con una placa con valores de referencia $Y=89,5 ; x=0,3176$ y y $=0,334$ ) para cada lote de salchichas se midieron las coordenadas CIELab en cinco puntos diferentes. Las mediciones de color se expresaron en términos de luminosidad $L^{*}\left(L^{*}=0\right.$ para el negro y $L^{*}=100$ para el blanco), y los parámetros de cromaticidad $a^{*}$ (verde [-] y rojo [+]) y $b^{*}$ (azul [-] y amarillo [+]). A partir de estos parámetros fueron calculadas las coordenadas $C$ (croma) y $h$ (tono) utilizando las ecuaciones 1 y 2 respectivamente. Igualmente, se midieron estos mismos parámetros as una salchicha comercial con el fin de tener un patrón de comparación.

$$
\begin{aligned}
& \mathrm{C}=\left(\mathrm{a}^{\star 2}+\mathrm{b}^{\star 2}\right)^{1 / 2} \\
& \mathrm{~h}=\arctan \left(\mathrm{b}^{\star} / \mathrm{a}^{\star}\right)
\end{aligned}
$$

\section{ANÁLISIS DE DATOS}

Los resultados obtenidos para coordenadas de color CIELab se expresan como la media aritmética de las 8 repeticiones llevadas a cabo para cada uno de los lotes. El nivel de significación para todos los tratamientos 
se determinó mediante ANOVA. La diferencia entre las medias de los tratamientos se identificó mediante la prueba de Tukey. Para realizar los análisis estadísticos se utilizó el software SPSS 16 para Windows $(p<$ $0,05)$.

\section{RESULTADOS Y DISCUSIÓN}

Los resultados indican que todos los parámetros de color se vieron afectados por la presencia del extracto oleoso de carotenoides totales. La adición del aceite pigmentado en todos los lotes de salchichas aumentó los valores de luminosidad $(L)$, coordenada $\mathrm{b}^{*}$, croma $(C)$ y tono $(h)$ y disminuyó el valor de la coordenada $\mathrm{a}^{*}$. Sólo la adición de $9 \mathrm{ml} / \mathrm{kg}$ aumentó el valor de la luminosidad, valores por encima de este volumen de extracto no generaron diferencias significativas. Considerando los resultados del parámetro tono $(h)$ todos los lotes son diferentes estadísticamente. Por su parte para la coordenada $a^{*}$, los lotes 2 y 3 son similares al igual que los lotes 5 y 6 ; y la coordenada $b^{*}$ y la croma (C) mostraron que solo los lotes 1 y 2 no son diferentes significativamente.

Los resultados de los parámetros de color para cada uno de los lotes se aprecian en la Figura 1. Las columnas de cada parámetro de color marcadas con la misma letra no son significativamente diferentes al nivel $0.05 \%$ de significación según la prueba de Tukey.

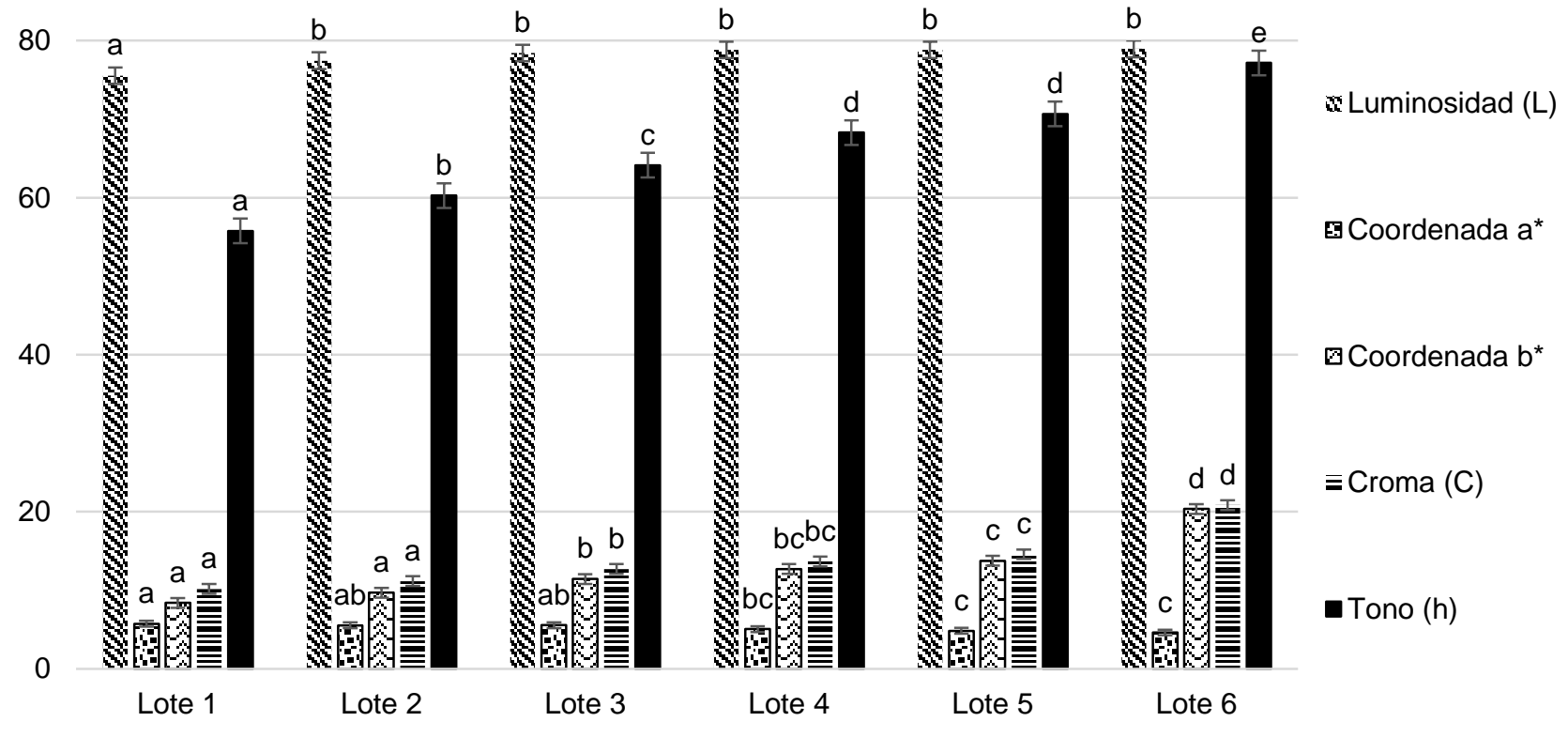

Fig. 1: Parámetros de color para cada uno de los lotes de las salchichas elaboradas.

La disminución en la cantidad de nitritos adicionados a cada lote causó un decrecimiento en el valor de la coordenada $\mathrm{a}^{*}$, mientras que el valor de luminosidad $(L)$ se incrementó. Los resultados para $L, \mathrm{a}^{*}, \mathrm{~b}^{*}$ y $h$ coinciden con lo encontrado por Isaza et al. (2012) al adicionar extracto de cereza en salchichas de carne de res y pasta de pollo encontrando un efecto ascendente significativo sobre los parámetros de color $L, b^{*}$ y tono $h$ y descendente para $a^{*}$ y croma $(C)$.

La disminución de la coordenada $\mathrm{a}^{*}$, conforme se disminuyó el contenido de nitritos y se aumentó la concentración del extracto de residuos de chontaduro, puede explicarse por la reducción del nitrito a óxido nítrico (NO) y su consecuente reacción con la mioglobina para formar nitrosomioglobina que se estabiliza por tratamiento térmico. A menor cantidad de nitritos, menor cantidad de reacciones formadoras de nitrosomioglobina. Estos resultados coinciden con los obtenidos por Deda et al. (2007) quienes trabajaron con salchichas de carne y cerdo adicionadas con nitrito de sodio y pasta de tomate.

De acuerdo a los estudios de Deda et al. (2007), cuanto más alto fue el nivel de pasta de tomate, mayores fueron los valores para la amarillez (coordenada $b^{*}$ ) y para la croma $(C)$, resultados que coinciden con los obtenidos en la presente investigación.

Otro estudio realizado por Amensour et al. (2010), quienes estudiaron la adición de extracto acuoso de hojas de Myrtus communis en salchichas de pollo tipo Frankfurt, los investigadores encontraron que los valores de luminosidad $(L)$ de las salchichas disminuyeron por la presencia del extracto y aumentó significativamente el 
valor de las coordenadas $a^{*}$ y $b^{*}$. Los resultados de luminosidad $(L)$ y coordenada $a^{*}$ son contrarios a los encontrados en este trabajo y los autores aducen el aumento de la coordenada $a^{*}$ a la posible presencia de compuestos con propiedades antioxidantes (fundamentalmente fenoles) en el extracto acuoso de Myrtus communis.

Amensour et al. (2010) explican que en los productos cárnicos la coordenada a* está influenciada por diversos factores tanto tecnológicos (emulsión en frío o caliente, tipo de picado, etc.) como de composición (relación fracción magra / fracción grasa, entre otros). Por su parte la luminosidad está relacionada con el agua libre en la superficie, el contenido de grasa, tejido conjuntivo y contenido y tipo de hemopigmentos. El comportamiento ascendente de la coordenada $b^{*}$ presentado en el estudio de Amensour et al. (2010) coincide con la adición de extracto de residuos de chontaduro y puede atribuirse a los carotenoides presentes en la harina de residuos de chontaduro que actúan incrementando el componente amarillo del color.

Eyiler y Oztan (2011) encontraron que el polvo de tomate adicionado a salchichas de res actuó como un agente colorante y acusó un aumento en el valor de la coordenada $\mathrm{a}^{\star}$ en los productos finales. Por el contrario, la luminosidad $(L)$ de las muestras disminuyó. La naturaleza de la materia prima utilizada en este estudio, con pigmentos rojizos les permitió obtener resultados contrarios a los observados con la adición de harina de residuos de chontaduro que presentan coloración cercana al naranja o amarillo. El aumento del valor del tono (h) muestra la disminución en el enrojecimiento de las salchichas (coordenada $a^{*}$ ), y el aumento en el amarilleo (coordenada $b^{*}$ ). Esta es la razón por la cual el tono $(h)$ de las salchichas adicionadas con harina de residuos de chontaduro aumentó coincidiendo con Eyiler y Oztan (2011) en la disminución de la coordenada $a^{*}$ y aumento de la coordenada $b^{*}$.

Hyun-Wook et al. (2015), haciendo uso del pigmento rojo del cártamo, colorearon fibra de trigo para elaborar seis tratamientos en salchichas cocidas con diferentes niveles de nitrito y dicha fibra. Al igual que en el presente estudio, la interacción de los niveles de nitrito y fibra coloreada afectaron significativamente todas las características de color de las salchichas. El aumento en el nivel de fibra coloreada resultó en una disminución de la luminosidad y el tono, pero aumentó el enrojecimiento, la amarillez y la croma. Resultados que coinciden con las salchichas coloreadas con el extracto oleoso de harina de residuos de chontaduro, desarrolladas en la presente investigación, en cuanto al aumento de la croma y la amarillez.

En el estudio de Hyun-Wook et al. (2015), el aumento en la coordenada b* podría estar relacionado con los cambios de color parciales de la cartamina presente en el cártamo durante el procesamiento térmico. En consecuencia, el procesamiento térmico podría convertirse en uno de los principales factores que afectan el aumento de la amarillez. Situación que de igual forma puede explicar el aumento del valor de la coordenada $b^{*}$ en las salchichas adicionadas con los carotenoides de los residuos del chontaduro después de ser sometidas a un tratamiento térmico.

Otro estudio desarrollado por González-Tenorio et al. (2013) en donde evaluaron la coloración de cuarenta variedades de chorizos comercializados en la zona centro de México, encontraron valores más altos para los parámetros luminosidad $(L)$ y las coordenadas $a^{*}$ y $b^{*}$ que los encontrados en la presente investigación. Probablemente, estos valores más altos se deben a las características de los productos analizados o a su formulación, ya que no se precisa cual fue el colorante o los colorantes utilizados en la fabricación de los mismos.

Las diferencias en el color entre las salchichas Frankfurt de diversos estudios están relacionadas con las características de color de los principales ingredientes utilizados en la elaboración de las mismas, según lo explica Isaza et al. (2012).

La Tabla 4 muestra la ANOVA y los resultados de la prueba de Tukey (letras diferentes en los superíndices de una misma columna indican diferencias significativas) para la comparación de medias entre los valores de las salchichas adicionadas con extracto oleoso de carotenoides de residuos de chontaduro y salchichas Frankfurt comerciales con el fin establecer la comparación con un producto de consumo habitual.

Los resultados obtenidos indican que ninguno de los lotes adicionados con el extracto es similar en cuanto a luminosidad y coordenada $\mathrm{a}^{*}$ (relacionada con el enrojecimiento) con las salchichas comerciales, lo cual probablemente es debido a la reducción de los nitritos. Los lotes adicionados con 9 y 19 ml de extracto oleoso son estadísticamente iguales, en cuanto a la coordenada b* (relacionado con el amarilleo) y la croma (C), al producto comercial. El extracto propuesto en esta investigación, al ser de color amarillo-anaranjado, proporciona en adiciones de 9 y $19 \mathrm{ml}$ la cantidad de color amarillo aceptado por el ocnsumidor. En cuanto al tono $(h)$, el lote con el mayor contenido de extracto oleoso $(97 \mathrm{ml})$ es similar al tono de las salchichas comerciales. 
Tabla 4: Comparación de los parámetros del color de las salchichas con extracto oleoso de carotenoides

\begin{tabular}{|c|c|c|c|c|c|c|c|}
\hline Lote & $\begin{array}{c}\text { Nitritos, } \\
\mathrm{mg} / \mathrm{kg}\end{array}$ & $\begin{array}{c}\text { Extracto, } \\
\mathrm{ml} / \mathrm{kg}\end{array}$ & $L^{1}$ & $\mathrm{a}^{*} 1$ & $\mathrm{~b}^{* 1}$ & $\mathrm{C}^{1}$ & $h^{1}$ \\
\hline 1 & 200 & 0 & $75,54 \pm 1,69^{\mathrm{b}}$ & $5,73 \pm 0,71^{\mathrm{a}}$ & $8,39 \pm 0,42^{\mathrm{a}}$ & $10,17 \pm 0,63^{\mathrm{a}}$ & $55,76 \pm 3,02^{\mathrm{a}}$ \\
\hline 2 & 180 & 9 & $77,45 \pm 1,71^{\mathrm{ab}}$ & $5,53 \pm 0,16^{\mathrm{ab}}$ & $9,69 \pm 0,35^{\mathrm{ab}}$ & $11,16 \pm 0,33^{\mathrm{ab}}$ & $60,26 \pm 0,99^{\mathrm{b}}$ \\
\hline 3 & 160 & 19 & $78,41 \pm 0,71^{\mathrm{a}}$ & $5,54 \pm 0,09^{\mathrm{ab}}$ & $11,44 \pm 0,28^{\mathrm{cd}}$ & $12,71 \pm 0,23^{\mathrm{bc}}$ & $64,13 \pm 0,83^{\mathrm{c}}$ \\
\hline 4 & 140 & 29 & $78,84 \pm 0,81^{\mathrm{a}}$ & $5,05 \pm 0,51^{\mathrm{ab}}$ & $12,70 \pm 0,33^{\mathrm{de}}$ & $13,67 \pm 0,18^{\mathrm{cd}}$ & $68,30 \pm 2,40^{\mathrm{d}}$ \\
\hline 5 & 120 & 42 & $78,82 \pm 0,88^{\mathrm{a}}$ & $4,83 \pm 0,39^{\mathrm{bc}}$ & $13,75 \pm 0,20^{\mathrm{e}}$ & $14,58 \pm 0,18^{\mathrm{d}}$ & $70,67 \pm 1,56^{\mathrm{d}}$ \\
\hline 6 & 100 & 97 & $78,95 \pm 0,53^{\mathrm{a}}$ & $4,62 \pm 0,31^{\mathrm{c}}$ & $20,34 \pm 2,15^{\dagger}$ & $20,85 \pm 2,16^{\mathrm{e}}$ & $77,17 \pm 0,63^{\mathrm{e}}$ \\
\hline Comercial & - & - & $69,00 \pm 5,29^{\mathrm{c}}$ & $2,73 \pm 1,24^{\mathrm{d}}$ & $11,00 \pm 1,00^{\mathrm{bc}}$ & $11,37 \pm 1,22^{\mathrm{ab}}$ & $76,38 \pm 5,40^{\mathrm{e}}$ \\
\hline
\end{tabular}

\section{CONCLUSIONES}

De los resultados obtenidos en este trabajo experimental se puede concluir que los aditivos naturales disueltos en aceites vegetales representan una alternativa para reducir el uso de nitritos en matrices alimenticias tipo cárnicas y favorecer al consumidor en sus preferencias naturales y saludables.

La adición del extracto oleoso pigmentado por los carotenoides de la harina de residuos de chontaduro en conjunto con la reducción del contenido de nitritos en las seis formulaciones elaboradas afectaron significativamente, a nivel estadístico, todos los parámetros de color de las salchichas Frankfurt.

Considerando solo la coordenada $\mathrm{b}^{*}$ para determinar el producto como aceptable por el consumidor en comparación con salchichas Frankfurt comerciales, puede determinarse que el contenido de nitrito permite ser reducido hasta en un 20\% utilizando $19 \mathrm{ml}$ del extracto oleoso de residuos de chontaduro por kilogramo de masa cárnica, sin embargo en general las salchichas pigmentadas naturalmente no logran obtener la coloración rojiza estándar comercial que se asume es la propiedad organoléptica aceptada y preferida por el consumidor.

\section{REFERENCIAS}

Aguilar, B, Utilización de residuo fibroso seco obtenido de la residuo de palmito de pejibaye (Bactris gasipaes H.B.K); en la elaboración de barras alimentarias energéticas, (BAE), en la industria Agrícola Exportadora, C.A INAEXPO, Tesis de Titulación, Facultad de Ciencia y Tecnología de Alimentos, Universidad Técnica de Ambato, Ambato-Ecuador (2010)

Amate, C.F. y otros cuatro autores, Development and validation of a LC-MS/MS method for the simultaneous determination of aflatoxins, dyes and pesticides in spices, Analytical and Bioanalytical Chemistry, 397(1), 93107 (2010)

Amensour, M. y otros siete autores, Estabilidad del color en salchichas de pollo tipo Frankfurt adicionadas con extracto acuoso de hoja de Myrtus communis. Óptica pura y aplicada, 43 (4), 251-257 (2010)

Andrée, S. y otros cuatro autores, Chemical safety of meat and meat products, Meat Science, 86 (1), 38-48 (2010)

Bazán-Lugo, E. y otros tres autores, Color compensation in nitrite-reduced meat batters incorporating paprika or tomato paste, Journal of the Science of Food and Agriculture, 92 (8), 1627-1632 (2012)

Bogantes, A., Manual de recomendaciones técnicas para el cultivo de palmito de pejibaye Bactris gasipaes H.B.K. Instituto Interamericano de Cooperación para la Agricultura Biblioteca Venezuela, Costa Rica. Ministerio de Agricultura y Ganadería de Costa Rica (2012)

Bolanho, B.C., E.D. Godoy y A.P. Beléia, Carbohydrate composition of peach palm (Bactris gasipaes Kunth) by-products flours, Carbohydrate Polymers 124, 196-200 (2015)

Boles, J.A. y R. Pegg., Meat color. In: The Saskatchewan Food Product Innovation Program, Montana State University and Saskatchewan Food Product Innovation Program University of Saskatchewan (2010), 4p, http://animalrange.montana.edu_courses_meat_meatcol. Acceso: 2 de febrero (2015) 
Chengaiah, B. y otros cuatro autores, Medicinal importance of natural dyes - a review, International Journal of PharmTech Research, 2 (1), 144 - 154 (2010)

Contreras. C. J, y otros tres autores, Antioxidant capacity, phenolic content and vitamin C in pulp, peel and seed from 24 exotic fruits from Colombia, Food Research International, 44 (7), 2047-2053 (2011)

Crews, C, Processing Contaminants: N-Nitrosamines, Encyclopedia of Food Safety, 2, 409-415 (2014)

Deda, M.S., J.G, Bloukas, y G.A, Fista, Effect of tomato paste and nitrite level on processing and quality characteristics of frankfurters, Meat Science, 76 (3), 501-508 (2007)

Eyiler, E. y A. Oztan, Production of frankfurters with tomato powder as a natural additive, Food Science and Technology, 44 (1), 307-311 (2011)

Fernández, C. y otros tres autores, Evaluación del contenido de licopeno en pastas de tomate comerciales, Información Tecnológica, 18 (3), 31-38 (2007)

Giraldo. A. y otros cinco autores, Estudio de la diversidad del chontaduro (Bactris gasipaes) consumido en Colombia, Revista de la Asociación Colombiana de Ciencia y Tecnología de Alimentos, 18 (edición especial), $1-6(2009)$

González-Tenorio, R. y otros tres autores, Caracterización de propiedades químicas y fisicoquímicas de chorizos comercializados en la zona centro de México, Información Tecnológica, 24 (2), 3-14 (2013)

Herrmann, S.S, K. Grandy y L. Duedahl-Olesen, Formation and mitigation of N-nitrosamines in nitrite preserved cooked sausages, Food Chemistry, 174, 516-526 (2015)

Hyun-Wook, K. y otros ocho autores, Wheat fiber colored with a safflower (Carthamus tinctorius L.) red pigment as a natural colorant and antioxidant in cooked sausages, LWT - Food Science and Technology, 64 (1), 350$355(2015)$

Isaza, Y.L., D.A. Restrepo y J.H. López, Efecto de la Inclusión de un Extracto de Cereza (Prunus avium L.) sobre el estado de Oxidación y las Características Fisicoquímicas y Sensoriales de Salchichas Tipo Frankfurt, Revista Facultad Nacional de Agronomía, 65 (1), 6541-6552 (2012)

Jatunov, S. y otros tres autores, Carotenoid composition and antioxidant activity of the raw and boiled fruit mesocarp of six varieties of Bactris gasipaes, Archivos Latinoamericanos de Nutrición, 60(1), 99-104 (2010)

Kuhnle, G.G.C. y S.A. Bingham, Meats, protein and cancer, in Bioactive Compounds and Cancer by J. Milner y D. Romagnolo, pp 195-212 Springer Science+Business Media, New York, USA (2010)

Kurcubic, V.S. y otros seis autores, Antioxidant and antimicrobial activity of Kitaibelia vitifolia extract as alternative to the added nitrite in fermented dry sausage, Meat Science, 97 (4), 459-467 (2014)

Larsson, S.C. y A. Wolk, Red and processed meat consumption and risk of pancreatic cancer: meta-analysis of prospective studies, British Journal of Cancer, 106, 603-607 (2012)

Leterme, P. y otros cinco autores, Chemical composition and nutritive value of peach palm (Bactris gasipaes Kunth) in rats, Journal of the Science of Food and Agriculture, 85 (9), 1505-1512 (2005)

Marchesi, C.M. y otros tres autores, Influência das condições de armazenamento sobre os pigmentos cárneos e a cor do salame italiano fatiado, Ciência e Tecnología Alimentar, 26 (3), 697-704 (2006)

Mercadante, A. Z. y otros tres autores, Effect of natural pigments on the oxidative stability of sausages stored under refrigeration, Meat Science, 84 (4), 718 - 726 (2010)

Moreira, I. y otros siete autores, Colour evaluation of a phycobiliprotein-rich extract obtained from Nostoc PCC9205 in acidic solutions and yogurt, Journal of the Science of Food and Agriculture, 92 (3), 598-605 (2012) NTC 1325: Instituto Colombiano de Normas Técnicas ICONTEC (Colombia), Industrias alimentarias, productos cárnicos procesados no enlatados, 1-32, Bogotá-Colombia (2008)

Ordóñez-Santos, L.E, L.X, Pinzón-Zarate, y L.O, González-Salcedo, Optimization of ultrasonic-assisted extraction of total carotenoids from peach palm fruit (Bactris gasipaes) by-products with sunflower oil using 
response surface methodology, Ultrasonics Sonochemistry, in press (2015)

Pangestuti. R y S.K, Kim, Biological activities and health benefit effects of natural pigments derived from marine algae, Journal of Functional Foods, 3 (4), 255 - 266 (2011)

Restrepo O. J., L.E, Vinasco y J.A. Estupiñán, Estudio comparativo del contenido de ácidos grasos en 4 variedades de Chontaduro (Bactris gasipaes) de la región del Pacífico Colombiano, Revista de Ciencias. Universidad del Valle, 16, 123-129 (2012)

Rohrmann, S, K. y otros cuarenta y seis autores, Meat consumption and mortality - results from the European Prospective Investigation into Cancer and Nutrition, BMC Medicine, 11, 63-75 (2013)

Rojas-Garbanzo, C. y otros tres autores, Identification and quantification of carotenoids by HPLC-DAD during the process of peach palm (Bactris gasipaes H.B.K.) flour, Food Research International, 44 (7), 2377-2384 (2011)

Sebranek, J.G. y J.N. Bacus, Cured meat products without direct addition of nitrate or nitrite: what are the issues? Meat Science, 77 (1), 136-147 (2007)

Solymosi, K. y otros tres autores, Food color additives of natural origin, Colour Additives for Foods and Beverages, 3-34 (2015)

Strati, F, I y Oreopoulou, V, Process optimization for recovery of carotenoids from tomato waste, Food Chemistry, 129 (3), 747-752 (2011)

Tamayo G. y J. Román, Estudio investigativo del chontaduro, análisis de sus propiedades, explotación y aplicación creativa en el ámbito culinario, Tesis de Titulación, Facultad de Turismo y Preservación Ambiental, Hotelería y Gastronomía, Universidad Tecnológica Equinoccial, Quito-Ecuador (2010)

Vargas, A.G. y C.J. Argüelles, Razas de palma de chontaduro (Bactris gasipaes H.B.K.) de acuerdo con las propiedades físico-químicas y bromatología del fruto, Instituto Amazónico de Investigaciones Científicas, SINCHI, 19 p, (2000)

Weiss, J. y otros tres autores, Advances in ingredient and processing systems for meat and meat products, Meat Science, 86 (1), 196-213 (2010) 
